\title{
Retrospective Analysis of Chest CT Imaging Findings of COVID-19 in Cancer Patients: Initial Experience in a Tertiary Cancer Center
}

\author{
Stathopoulos $\mathrm{K}^{1 *}$, Bali AM${ }^{1}$, Emonts $\mathrm{P}^{1}$, Loizidou $\mathrm{A}^{2}$, Lefebvre $\mathrm{Y}^{1}$, Picchia $\mathrm{S}^{1}$ \\ and Lemort $\mathbf{M}^{1}$ \\ ${ }^{1}$ Departement of Radiology, Institute Jules Bordet- ULB, Belgium \\ ${ }^{2}$ Departement of Internal Medicine, Institute Jules Bordet- ULB, Belgium
}

*Corresponding author: Dr Konstantinos Stathopoulos, Institut Jules Bordet, 1 Heger Bordet str, 1000, Brussels, Belgium, Tel: 0032471259729; 003225413989; Email: konstantinos. stathopoulos@bordet.be

\section{Research Article}

Volume 4 Issue 3

Received Date: August 04, 2020

Published Date: September 07, 2020

DOI: $10.23880 /$ crij- 16000176

\section{Abstract}

Purpose: To assess the diagnostic value of initial chest CT findings and the CT-based severity scoring system to predict the clinical outcome of COVID-19 in cancer patients.

Materials and Methods: Between March 1st and May 15th 2020, we included all consecutive cancer patients (with hematological or solid malignancy) with clinical suspicion of COVID-19, or asymptomatic patients admitted for cancer treatment and screened for COVID-19. Diagnostic performance of chest CT for diagnosis and disease severity assessment was evaluated. Disease severity was evaluated based on the SFR (Societé Française de Radiologie) scoring system. Association between chest CT severity score and clinical outcome was also assessed.

Results: Amongst 197 consecutive cancer patients, 46 were confirmed COVID-19 with available initial chest CT (24 men and 22 women; median age of 67 years). 20/46 had a haematologic disease and 26/46 had a solid malignancy. Sixteen (35\%) patients were suspected to have hospital-associated transmission. Common chest CT findings were pure ground-glass opacity (GGO) (39/46, 85\%) followed by GGO with consolidation (19/46-41\%), pure consolidation (16/46-35\%) and crazy paving (16/46-35\%). Based on RT-PCR test as standard of reference, chest CT demonstrated $96 \%$ sensitivity, $82 \%$ specificity, $62 \% \mathrm{PPV}$, $98 \% \mathrm{NPV}$ and $85 \%$ accuracy for the diagnosis in the whole population. A total of $19 / 46$ (41\%) patients had severe events and the mortality rate was $35 \%$ (16/46), 22\% (10/46) COVID-related. There was a significant association between the number of lobe involved and the need for hospitalisation, poor progression or death ( $\mathrm{p}=0.04)$, and between chest CT scoring system and survival ( $\mathrm{p}=.0005)$.

Conclusion: The high performance for diagnosis and severity assessment for COVID-19 suggest to use chest CT to identify infected cancer patients and to early isolate them to avoid nosocomial contamination both for cancer patient and healthcare staff.

Keywords: COVID-19; SARS-CoV-2; Chest CT; Cancer; Retrospective case study

Abbreviations: SARS: Severe Acute Respiratory Syndrome; MERS: Middle East Respiratory Syndrome; WHO: World Health Organization; FBP: Filtered-Back-Projection;
DLP: Dose-Length-Product; PACS: Picture and Archiving Computer System. 


\section{Clinical Radiology \& Imaging Journal}

\section{Introduction}

Severe Acute Respiratory Syndrome CoronaVirus 2 (SARS-CoV-2) is a novel enveloped RNA beta-coronavirus belonging to the same family of viruses causing severe acute respiratory syndrome (SARS) and Middle East respiratory syndrome (MERS) [1]. First described in November 2019 in Wuhan, China, the disease was named Coronavirus Disease 2019 (COVID-19). The virus has spread rapidly across the globe and COVID-19 was declared pandemic by the World Health Organization (WHO) on the 11th of March 2020. Up to date there are no proven treatments for COVID-19 and current management of the pandemic mainly lies on limiting transmission of the disease by early detection and isolation of patients.

Reverse Transcriptase Polymerase Chain Reaction for coronavirus (RT-PCR SARS-COV -2) represents the standard of reference for the rapid detection of the virus and the diagnosis of COVID-19 with very high specificity (93\%) but with variable sensitivity $(50-100 \%)$ [2]. The test is performed on naso-pharyngeal swab sampling [3]. False negative results seem to be associated with several variables such as the viral load kinetics in different anatomic sites of the patients, sampling procedures, inappropriate collection, transportation, handling or even the presence of amplification inhibitors in the sample. One negative RT-PCR test therefore does not exclude COVID-19 and multiple repeation of the test may be required to make the final diagnosis, leading to multiple days of uncertainty for both patients and healthcare professionals.

In clinical suspicious cases for COVID 19 combination of real-time RT-PCR SARS -COV 2 and CT features could facilitate disease diagnosis and management. Therefore, radiological findings play an important role in this context and our knowledge about the imaging features of the associated pneumonia is rapidly evolving $[4,5]$ The clinical spectrum of COVID-19 pneumonia is broad and includes mild to more severe and critical cases. [6-8]. Furthermore, CT imaging could contribute to clinical diagnosis and illness assessment by flagging suspected cases, grading the severity and non-invasively evaluating disease evolution [9].

There remains a great deal of uncertainty about COVID-19 and its effects on individuals, especially those immunocompromised and with multiples comorbidities. Patients with cancer represent a unique subset of subjects who are often both elderly and immunocompromised, may have substantial comorbidities and may be receiving treatment that several times affect immunity increasing the risk of concomitant infections. Given the prevalence of cancer worldwide and the high transmissibility of COVID-19, there is a pressing need to understand the effects of this new viral infection and its associated and potentially serious outcomes specifically for cancer patients [10].

This brings to the aim of the present study which was to evaluate the value of initial chest CT findings and CT-based severity scoring systems to predict the clinical outcome of COVID-19 in cancer patients.

\section{Materials and Methods}

This is a monocentric (cancer center) retrospective study which received approval from the local ethical committee and patient consent was waived.

\section{Patient Population}

Between March $1^{\text {st }}$ and May $15^{\text {th }} 2020$, consecutive cancer patients (presenting hematologic or solid malignancy) with clinical suspicion of COVID-19 pneumonia or asymptomatic patients with hematological malignancy admitted for cancer treatment and screened for COVID-19, who underwent to RTPCR and low dose chest CT, were considered.

Among these cancer patients, those with confirmed COVID-19 based on at least one positive RT-PCR test result for SARS-CoV-2 and at least one chest CT scan showing lung abnormalities were included. No specific exclusion criteria were applied.

\section{Clinical Data}

Demographic variables collected included patient age and gender, cancer type and disease stage, treatment type, infection source, length from symptom onset to admission, admission unit, interval between consecutive follow up chest CT scan and mortality rate.

\section{CT Scan Acquisition Protocol}

Unenhanced low dose chest CT scans were acquired using either a 192 slice SIEMENS Somatom FORCE Dual Energy CT scanner (Siemens Healthineers, Forchheim, Germany) or using a 40slice SOMATOM Definition CT scanner (Siemens Healthineers, Erlangen, Germany). Patients were in supine position and scanning was performed at full inspiration. To minimize motion artifacts, patients were instructed on breath-holding. The scanning field of view was set from the level of thoracic inlet to the lowest costophrenic angle.

The acquisition and reconstruction parameters were as follows: $120 \mathrm{kV}$ tube voltage with automatic tube current modulation (100-350 mAs), $1 \mathrm{~mm}$ slice thickness without interslice gap, a collimation of $0.625 \mathrm{~mm}$ or $0.5 \mathrm{~mm}$, a pitch of 0.8 or 1 and matrix of $512 \times 512$ using filtered-backprojection (FBP) reconstruction (SOMATOM Definition) 


\section{Clinical Radiology \& Imaging Journal}

or using an advanced modeled iterative reconstruction (strength 3) using Br40 (mediastinal) and BI57 (lung) kernels (Somatom Force, Siemens Healthineers). The mediastinal and lung window width and level were set as $400 / 40 \mathrm{HU}$ and 1500/-500(Siemens Healthineers) respectively to evaluate the abnormalities in the mediastinum and lung parenchyma. The median dose-length-product (DLP) was $39 \mathrm{mGycm}$ (range 15-147 mGycm) for Somatom Force and $143 \mathrm{mGycm}$ (range 60-218 mGycm) for Somatom Definition.

\section{CT Images Analysis}

All chest CT images were independently reviewed by one thoracic radiologist with 18 years of experience and blinded to the clinical and biological data using a picture and archiving computer system (PACS). CT findings characteristics are summarized in table 1.

\begin{tabular}{|c|c|}
\hline CT distribution and pattern & Classification \\
\hline \multirow[t]{2}{*}{ Lobe distribution } & Right lung: Upper / Middle / Lower \\
\hline & Left Lung : Upper /Lower \\
\hline \multirow[t]{2}{*}{ Pulmonary distribution pattern } & Central / Peripheral / Mixed \\
\hline & Anterior / Posterior / Mixed \\
\hline \multirow[t]{8}{*}{ Lesion Morphology-typical patterns } & Crazy paving \\
\hline & GGO with consolidation \\
\hline & Intralobular septal thickening \\
\hline & Interlobular septal thickening \\
\hline & Linear opacities \\
\hline & Pure Ground-glass opacities (GGO) \\
\hline & Reticulation \\
\hline & Reversed halo sign \\
\hline \multirow{9}{*}{ Lesion morphology-other patterns } & Consolidation with air bronchograms \\
\hline & Consolidation (Pure) \\
\hline & Emphysema/COBP \\
\hline & Lung calcification \\
\hline & Lung cavitation \\
\hline & Lymphadenopathy \\
\hline & Mosaic attenuation \\
\hline & Pericardial effusion \\
\hline & Pleural effusion (Bilateral / Left / Right) \\
\hline
\end{tabular}

Table 1: CT findings characteristics.

Multiple GGO (GGO), bilateral/multifocal involvement, peripheral distribution, crazy paving, consolidation, and reversed halo sign were consistent of COVID-19. The identification of tree-in-bud opacities, centrilobular / peribronchovascular distribution, cavitation and pleural effusion were considered chest CT findings inconsistent of COVID-19 [11].

Two diagnostic and one scoring system were applied: 1. French Society of Radiology (SFR) diagnostic and scoring system, which included [12]

a. Three categories of probability of COVID-19 pneumonia: typical, consistent and inconsistent;

b. Five-point score grading system to estimate the extent of pulmonary involvement ranging from 0 to 5 : score $0,0 \%$ involvement; score 1, 1-10\% involvement; score 2, 11$25 \%$ involvement; score 3, 26-50\% involvement; score
4, 51-75\% involvement, score 5, 76-100\%.

2. ACR/Fleischner Society: [13]

Four categories of probability of COVID-19 pneumonia:

a. Typical features [14-19]

b. Indeterminate features (reported in COVID-19 pneumonia but are not specific enough)

c. Atypical features (reported to be uncommon or not occurring in COVID-19 pneumonia; more typical of other diseases)

d. Negative features for pneumonia (no parenchymal abnormalities that could be attributable to infection). Importantly, there may be no findings on early $\mathrm{CT}$ in COVID-19.

\section{Theory/Calculation (Statistical Analysis)}

For descriptive analysis, continuous variables were 


\section{Clinical Radiology \& Imaging Journal}

presented as average and standard deviation or as median with interquartile range (IQR), as appropriate. Categorical variables are presented as number and percentage. Statistical analyses were performed using MedCalc Software (Ostend, Belgium). Chi-square test was performed to assess association between number of involved lung lobes on initial chest CT, and patient outcome. Based on RT-PCR as standard of reference, sensitivity, specificity, positive predictive value (PPV), negative predictive value (NPV) and accuracy of chest CT were calculated based on SFR and ACR/ Fleischner Society diagnostic systems. Wald chi square tests were performed to access association between SFR severity score of initial chest $\mathrm{CT}$, age, with patient survival, estimated at the date of the end of the study. A p-value of less than 0.05 (two-tailed) was considered statistically significant.

\section{Results}

A total of 197 patients underwent to RT-PCR test and chest CT scan. 61/197 (31\%) had negative RT-PCR test and negative CT findings. 72/197 (37\%) had negative RTPCR test and atypical CT findings for COVID-19 pneumonia. 46/197 (23\%) presented positive RT-PCR test and positive CT findings for COVID-19 pneumonia. Table 2 reports demographic and clinical characteristics of these 46/197 patients. Lung and breast cancer were the most frequent types of cancer (6/46). 14/46 patients had active metastatic disease.

\begin{tabular}{|c|c|c|c|}
\hline Age & & Patients $(\mathrm{N}=46)$ & $\%$ \\
\hline Age-median-years(MIN-MAX)-years & 67yo (38-83) & & \\
\hline \multicolumn{4}{|c|}{ Gender } \\
\hline Male & & 24 & $52 \%$ \\
\hline Female & & 22 & $48 \%$ \\
\hline \multicolumn{4}{|l|}{ TUMOUR TYPE } \\
\hline Haematological Malignancies & & 20 & $44 \%$ \\
\hline Non-Hodgkin Lymphoma & & 7 & \\
\hline Acute Myeloid Leukemia & & 4 & \\
\hline Multiple myeloma & & 4 & \\
\hline Chronic Myeloid Leukemia & & 2 & \\
\hline DLBCL & & 1 & \\
\hline Lymphoid Chronic Leucemia & & 1 & \\
\hline Hodgkin Lymphoma & & 1 & \\
\hline Solid malignancies & & 26 & $56 \%$ \\
\hline Lung cancer & & 6 & \\
\hline Breast cancer & & 6 & \\
\hline Ovarian serous adenocarcinoma & & 3 & \\
\hline Cervical cancer & & 3 & \\
\hline Renal cancer & & 2 & \\
\hline Urothelial cancer & & 2 & \\
\hline Prostate cancer & & 1 & \\
\hline Oesophageal cancer & & 1 & \\
\hline Hepatocellular carcinoma & & 1 & \\
\hline Gastric cancer & & 1 & \\
\hline Colorectal cancer & & 1 & \\
\hline \multicolumn{4}{|l|}{ ONGOING ANTI-CANCER TREATMENT } \\
\hline Chemotherapy ( $<14$ days) & & 15 & $33 \%$ \\
\hline Target/immunotherapy & & 12 & $24 \%$ \\
\hline Chemo/radiotherapy & & 11 & $26 \%$ \\
\hline Immunotherapy (<14 days) & & 4 & $9 \%$ \\
\hline Target therapy $(<14$ days $)$ & & 4 & $9 \%$ \\
\hline
\end{tabular}

Table 2: Demographic and clinical characteristics of covid-19-infected cancer patients. 


\section{Clinical Radiology \& Imaging Journal}

43/46 patients (94\%) had positive RT-PCR for COVID-19 at symptoms onset and 46/46 patients (100\%) had any positive RT-PCR test (1-6 days after onset of symptoms). The median time from initial disease onset to RT-PCR testing was 4.2 days (range 0-26 days). The median time from initial disease onset to CT scan was 4.9 days (range 0-21 days); Two source of infection were identified: 16/46 patients (35\%) developed COVID-19 while undergoing antitumor therapy in hospital and 30/46 patients (65\%) in their communities. Furthermore, 5/46 patients (11\%) were asymptomatic, $19 / 46$ patients (41\%) developed severe radiological and clinical events. 39/46 (85\%) were admitted for hospitalisation. 10/39 (26\%) were admitted either to ICU or to High-Dependency-Unit (HDU).

At the end of the study (15 ${ }^{\text {th }}$ May 2020), 13/46 (28\%) patients had totally recovered, $17 / 46$ (37\% patients were still hospitalised and 16/46 patients (35\%) died. 10/16 deaths were COVID-19 related (10/46 patients-22\%). The cause of COVID-19 related death included ARDS (6/10), multi-organic failure $(2 / 10)$, pulmonary embolism $(1 / 10)$ and septic shock (1/10). 44/46 patients had abnormal findings on initial chest CT. Table 3 reports the pattern and distribution of the observed chest CT abnormalities. Pure GGOs opacities were the most common feature $(39 / 46$, $85 \%)$ followed by GGO with consolidation $(19 / 46,41 \%)$ and pure consolidation (16/46-35\%). The number of lobe involved ( $n=0-5$ lobes) was significantly associated with the need for hospitalisation, poor progression or death $(\mathrm{p}=0.04)$. Lymphadenopathy was observed in 6/46 patients (13\%) but related with cancer (on the basis of previous CT exams) and not to COVID-19 pneumonia.

Pulmonary nodules were found in $7 / 46$ patients but there was no relation with COVID-19 on the basis of previous CT exams (lung metastasis). In 1 patient, cavitation was observed which was present on previous CT scans and highly suggestive of aspergilloma. Other abnormalities, such as pericardial effusion, lung calcification and pneumothorax were totally absent.

\begin{tabular}{|c|c|c|c|}
\hline Patterns and distribution & Localisation & Patients $N=46$ & $\%$ \\
\hline \multirow[t]{6}{*}{ Pulmonary distribution pattern } & Central & 5 & 11 \\
\hline & Peripheral & 31 & 70 \\
\hline & Mixed & 8 & 18 \\
\hline & Anterior & 4 & 9 \\
\hline & Posterior & 31 & 68 \\
\hline & Mixed & 11 & 24 \\
\hline \multirow[t]{3}{*}{ Laterality of lung lobe involvement } & Bilateral & 33 & 72 \\
\hline & Left & 3 & 7 \\
\hline & Right & 8 & 18 \\
\hline \multicolumn{4}{|c|}{ Lesion Morphology-typical patterns } \\
\hline Crazy paving & & 16 & 36 \\
\hline \multirow[t]{6}{*}{ GGO with consolidation } & $0 / 5$ lobes & 27 & 61 \\
\hline & $1 / 5$ lobes & 2 & 5 \\
\hline & $2 / 5$ lobes & 3 & 7 \\
\hline & $3 / 5$ lobes & 7 & 16 \\
\hline & 4/5 lobes & 4 & 9 \\
\hline & 5 lobes & 3 & 7 \\
\hline Intralobular septal thickening & & 18 & 41 \\
\hline Interlobular septal thickening & & 15 & 34 \\
\hline Linear opacities & & 2 & 5 \\
\hline Pure Ground-glass opacities (GG0) & $1 / 5$ lobe & 8 & 18 \\
\hline
\end{tabular}




\section{Clinical Radiology \& Imaging Journal}

\begin{tabular}{|c|c|c|c|}
\hline & 2/5 lobes & 6 & 14 \\
\hline & $3 / 5$ lobes & 7 & 16 \\
\hline & 4/5 lobes & 8 & 18 \\
\hline & $5 / 5$ lobes & 10 & 22 \\
\hline Reticulation & & 9 & 20 \\
\hline Reversed halo sign & & 0 & 0 \\
\hline Lesion morphology-other patterns & & & \\
\hline Consolidation (pure) & & 16 & 36 \\
\hline Consolidation with air bronchograms & & 4 & 9 \\
\hline Emphysema/COBP & & 17 & 38 \\
\hline Lymphadenopathy & & $6 *$ & 14 \\
\hline Lung cavitation & & $1 *$ & 2 \\
\hline Lung calcification & & 0 & 0 \\
\hline Mosaic attenuation & & 0 & 0 \\
\hline Pericardial effusion & & 0 & 0 \\
\hline Pleural effusion & & 10 & 22 \\
\hline Pleural effusion-laterality & Bilateral & 8 & 18 \\
\hline & Left & 1 & 2 \\
\hline & Right & 1 & 2 \\
\hline *Non-COVID-19 related & & & \\
\hline
\end{tabular}

Table 3: Findings on initial CT.

Table 4 reports the SFR scoring system for the parenchymal involvement on the initial chest CT scan and at follow up. The SFR scoring system calculated on initial chest CT showed a significant association with overall survival $(\mathrm{p}=.0005)$. The SFR scoring system calculated on initial chest CT and its association with the age of the patients didn't show a significant association with overall survival $(\mathrm{p}=0.0679)$. CT scans demonstrated excellent sensitivity, specificity, PPV, NPV, and accuracy for the diagnosis of COVID-19 pneumonia. The SFR probability system reported 96\%, 82\%, 62\%, 98\%, and $85 \%$ and the ACR/Fleischner Society probability system reported $96 \%, 88 \%, 71 \%, 99 \%$, and $90 \%$, respectively.

\begin{tabular}{|l|c|c|c|c|c|c|c|}
\hline \multicolumn{9}{|c|}{ SFR SCORING SYSTEM on initial CT and FUs } \\
\hline & & INITIAL CT & FU1 & FU2 & FU3 & FU4 & FU5 \\
\hline & CTs(n) & 46 & 25 & 16 & 7 & 2 & 1 \\
\hline \multirow{4}{*}{ SFR SCORES } & 0 & $2(4 \%)$ & 0 & 0 & 0 & 0 & 0 \\
\cline { 2 - 9 } & 1 & $22(48 \%)$ & $8(32 \%)$ & $3(19 \%)$ & 0 & 0 & 0 \\
\cline { 2 - 9 } & 2 & $11(24 \%)$ & $3(12 \%)$ & $1(6 \%)$ & 0 & 0 & 0 \\
\cline { 2 - 9 } & 3 & $6(13 \%)$ & $5(20 \%)$ & $4(25 \%)$ & $2(29 \%)$ & 0 & 0 \\
\cline { 2 - 9 } & 4 & $5(11 \%)$ & $8(32 \%)$ & $3(19 \%)$ & $3(43 \%)$ & 0 & 0 \\
\cline { 2 - 9 } & 5 & 0 & $1(4 \%)$ & $5(32 \%)$ & $2(29 \%)$ & 2 & 1 \\
\hline
\end{tabular}

Notes - CT: Computed Tomography/ FU: Follow-up/SFR: French Society of Radiology)

Table 4: SFR SCORING SYSTEM on initial CT and FUs. 


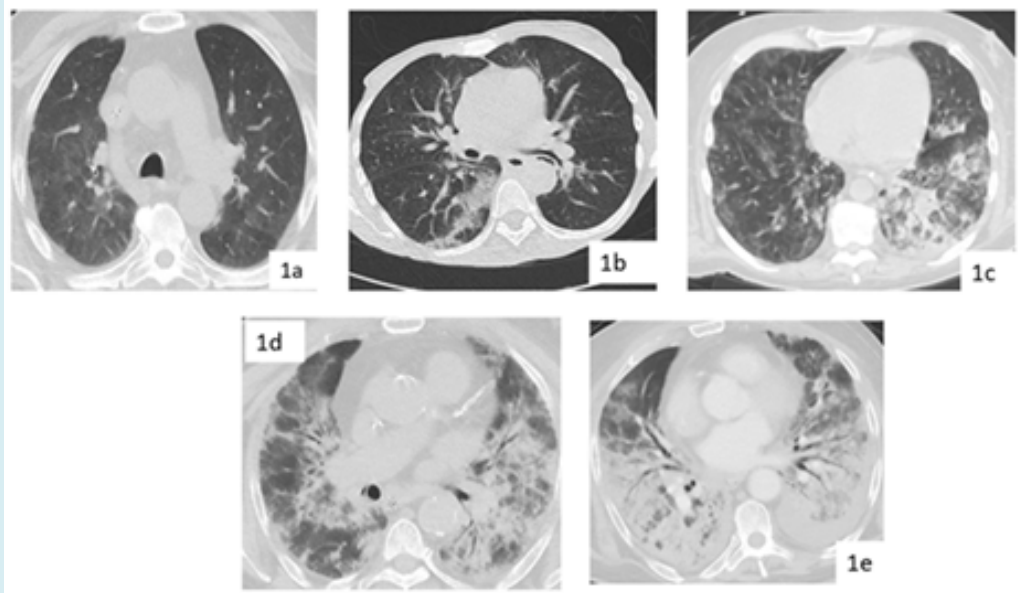

Figure 1: Chest CT imaging features for COVID-19 with different parenchymal severity SFR scores- 1a: Typical chest CT imaging features for COVID-19 with posterior, peripheral and rounded pure GGO in axial images of a haematological cancer patient (SFR score 1); 1b: Indeterminate CT imaging features for COVID-19. Unenhanced axial images showing patchy GG0 with nonrounded morphology and no specific distribution, in a case of COVID-19 (score SFR 2); 1c: Typical chest CT imaging features for COVID-19 with pure bilateral mixed (peripheral and central) GGO with crazy paving and consolidation with air bronchogram of the left inferior lobe in a haematological cancer patient ( SFR score 3); 1d-1e: posterior and anterior, peripheral and central bilateral and multiple GGOs and patchy consolidations with air bronchograms; Organising pneumonia and influenza pneumonia can be indistinguishable from COVID-19 (SFR scores 4 and 5 respectively).

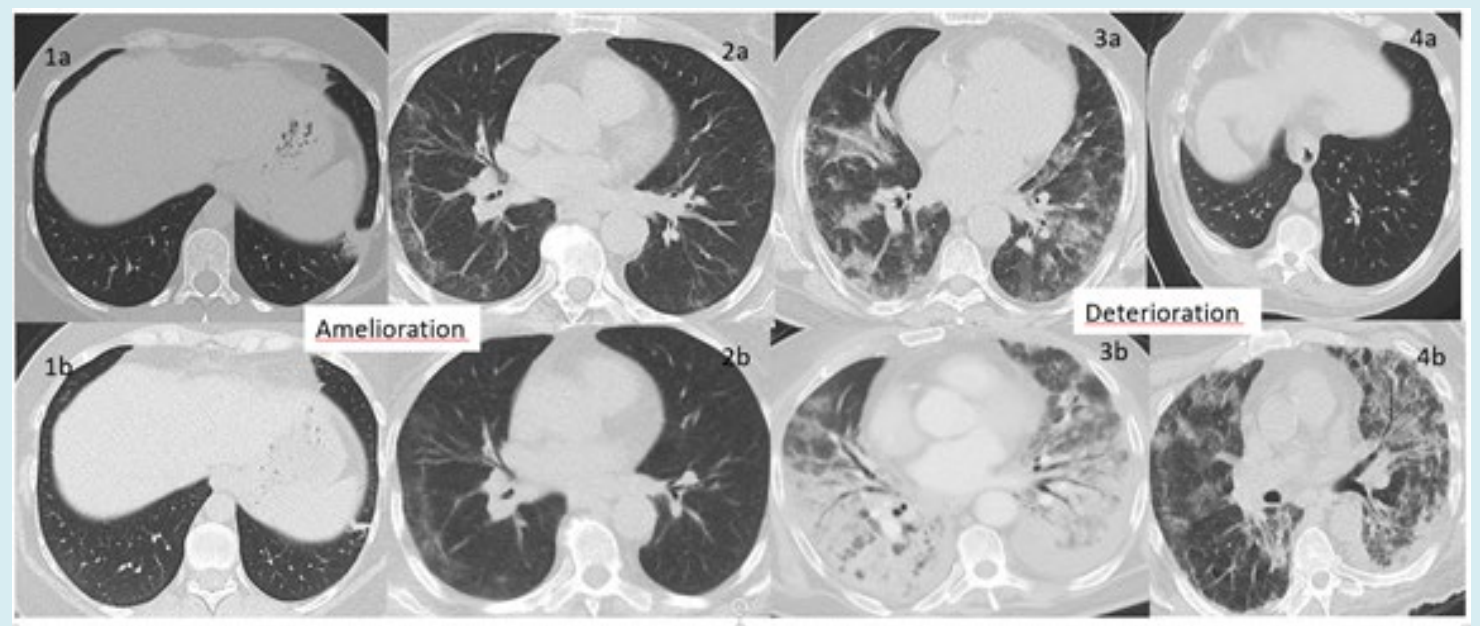

Figure 2: Two patients with radiological amelioration (1a-1b and 2a-2b) and two patients with radiological deterioration (3a$3 \mathrm{~b}$ and $4 \mathrm{a}-4 \mathrm{~b}$ ) between the initial chest CT and the follow-up 1. All patients were symptomatic with positive RT-PCR SARS-COV -2 on admission.

The average time interval between the initial CT and the FU1 was 12.4 days (SD 8.3 days), between the initial CT and FU2 22.6 days and between the initial CT and the FU3 24.3 days (SD 15.5 days). Furthermore, the average time between FU1-FU2 was 9.6 days and FU2-FU3 was 12 days. At first FU 18/46 (39\%) patients presented a deterioration of radiological CT findings, 3/46 (6\%) an improvement, 4/46
(9\%) patients a relatively unchanged appearance. 21/46 (46\%) patients did not have a FU due to clinical stability or improvement. At the FU2, 16/46 patients underwent chest CT FU and 9/16 (56\%) patients presented a deterioration of radiological CT findings, 4/16 (25\%) a radiological improvement, $3 / 16(9 \%)$ a relative unchanged appearance. At FU3 only 7/46 (\%) patients had available chest CT and 
$3 / 7(43 \%)$ patients presented a deterioration and $4 / 7$ (57\%) an improvement.

\section{Discussion}

Since the beginning of COVID-19 pandemic, older population and patients with non-communicable diseases (NCDs), including cancer, have been reported to be at increased risk of COVID-19, of severe complications and poor outcomes [4,8,16,20-23]. Cancer patients with COVID-19 still represents a poor studied population [24-27].

Early diagnosis of COVID-19 is crucial for disease control and treatment. Compared to RT-PCR, chest CT may be a more reliable, practical and on top of all a rapid objective method to diagnose and evaluate COVID-19 pneumonia especially in this sensitive and fragile patient population.

In the present study, 23\% (46/197) of all tested cancer patients for COVID-19 showed positive chest CT findings. Furthermore, 94\% (43/46) of patients confirmed by RTPCR assays showed positive findings on chest CT which was higher than that reported by Zu, et al. [28] but consistent with what reported by Xie, et al. [29]. 35\% (16/46) of our patients developed COVID-19 infection during hospitalisation (no visits of family or relatives were allowed) and therefore nosocomial transmission was highly suspected. This is consistent with the previous study of Zhang et al in which a rate of $29 \%$ was reported [21].

We observed severe events in $41 \%$ of our population presenting severe radiological findings (defined by SFR score $\geq 3$ ) and admitted either to ICU or to HDU. Our results are in the same range of previous study [16].

The mortality rate in our study was $35 \%(16 / 46)$ but COVID-19-related mortality rate was $22 \%(10 / 46)$ which again is in the same range than previous studies $[17,18]$.

Consistently with previous studies, the predominant radiological patterns on initial CT scans in our patients were GGO's with peripheral posterior distribution and bilateral, multifocal lower lung involvement [20-23]. Some radiologic features were more common in severe cases, such as crazypaving pattern, consolidation with air bronchograms and pleural effusion compared to mild cases. In line with other studies on non-oncological patients, we noticed that severe cases in our cancer population usually presented a large lobar or bilobar consolidation with diffuse appearance indicating that the volume and extension of GGOs could also be considered an important factor of disease severity. As a matter of fact we found a statistically significant association between number of involved lung lobes and clinical outcome.
In the majority of severe cases either on the initial CT or on the first chest CT follows up when clinical worsening of the disease was observed, there was an increase of GGO with consolidation. On the other hand, pure GGOs decreased. Furthermore, consistent with previous studies on non-cancer patients and with the results of Pan et al the predominant GGO in early stage of the disease, was followed by development of crazy paving and finally, increasing consolidation later in the disease evolution [30,31].

We also observed that the severity of lung involvement on the initial chest CT was associated with need for hospitalisation, poor progression and unfavourable COVID-19 outcome (hospitalisation in ICU or death). The involvement of at least three lung lobes together with $>25 \%$ lung parenchyma implication (SFR scores 3-5) independently of the cancer type could be considered imaging criteria predictive of poor clinical outcome during COVID-19 course. In our study population 11 patients presented these criteria, 6 of which were diagnosed with haematological malignancy and all these patients died.

Previous studies in non-oncologic have reported a significant association between patient age and COVID-19 clinical outcome, with elderly patients showing worse disease course $[10,26]$. Our results appear to be discordant since in our cancer population, age seems not to be an unfavourable factor of disease evolution.

With respect to the diagnostic performance of chest CT for the diagnosis of COVID-19, we showed excellent results for both disease diagnosis and severity scoring systems in accordance with previous reported studies [30]. Thus the high sensitivity of chest CT provides a quick objective diagnostic test to assess the presence of lung disease secondary to SARS-CoV-2 infection. Furthermore, chest radiological manifestations together with the results of RTPCR tests may be considered imperative in the early detection and assessment of the severity of SARS-CoV-2 pneumonia.

Moreover some cancer patients in our study developed COVID-19 during hospitalisation whilst receiving antitumor treatment. However, treatment postponement in cancer patients should not be considered a reasonable choice to contain the infection risk in the ongoing pandemic. Treatment decision-making must be considered on case-bycase evaluation, aiming to meet patient safety and the risk of oncologic disease progression with an important impact on patient outcome [32].

Thus, quick recognition of the radiological appearances is imperative for early detection and assessment of the severity of COVID-19 and for the safety of both cancer patients and healthcare staff. Moreover, there is currently no evidence and 


\section{Clinical Radiology \& Imaging Journal}

no clear recommendations on whether stopping or delaying anti-cancer treatment including conventional cytotoxic chemotherapy, immunotherapy and radiation therapy, in palliative but also in adjuvant settings, as well as the use of supportive care such as hematopoietic growth factors, would be advisable for patients with cancer.

Our study has several limitations. First, this retrospective study is based on a relatively limited number of patients, the probability of selection bias and the availability of data especially for the long term- hospitalised patients, could be of concern. Some important parameters such as tumour stage were not able to be included in the multivariate analyses. Patients included presented different tumour types so population heterogeneity could not be avoided. Longterm chest CT follow-up are needed to better understand the development of the disease. Thus, future prospective study designed with larger sample sizes could better explore the risk factors of radiological and clinical severity in COVID-19cancer patients.

\section{Conclusion}

Chest CT scan may be a reliable, highly available and rapid method to diagnose and assess severity of COVID-19. Suspicious CT scan may allow early isolation of the cancer patients until confirmation of the disease by RT-PCR SARSCOV 2 test, to contain nosocomial contamination both for cancer patient, in particular those affected by haematological disease, and healthcare staff safety.

\section{References}

1. CDCP (2019) Corona virus 2019 disease (COVID-19). Centers for Disease Control and Prevention.

2. Kim H, Hong H, Yoon SH (2020) Diagnostic Performance of CT and Reverse Transcriptase-Polymerase Chain Reaction for Coronavirus Disease 2019: A Meta-Analysis. Radiology 296(3).

3. Visseaux B, Hingrat QL, Collin G, Bouzid D, Lebourgeois S, et al. (2020) Evaluation of the QIAstat-Dx Respiratory SARS-CoV-2 Panel, the first rapid multiplex PCR commercial assay for SARS-CoV-2 detection. J Clin Microbiol 58(8).

4. Yang R, Li X, Liu H, Zhen Y, Zhang X, et al. (2020) Chest CT Severity Score: An Imaging Tool for Assessing Severe COVID-19. Radiology: Cardiothoracic Imaging 2(2).

5. Dangis A, Gieraerts C, Bruecker YD, Janssen L, Valgaeren $\mathrm{H}$, et al. (2020) Accuracy and reproducibility of low-dose submillisievert chest CT for the diagnosis of COVID-19. Radiology: Cardiothoracic Imaging 2(2).
6. Zhou S, Zhu T, Wang Y, Xia L (2020) Imaging features and evolution on CT in 100 COVID-19 pneumonia patients in Wuhan, China. European radiology 2020.

7. Xiong Y, Sun D, Liu Y, Yanqing F, Lingyun $Z$, et al. (2020) Clinical and High-Resolution CT Features of the COVID-19 Infection: Comparison of the Initial and Follow-up Changes. Invest Radiol 55(6): 332-339.

8. Kanne JP (2020) Chest CT Findings in 2019 Novel Coronavirus (2019-nCoV) Infections from Wuhan, China: Key Points for the Radiologist. Radiology 295(1): 16-17.

9. Wang Y, Dong C, Hu Y, Li C, Ren Q, et al. (2020) Temporal Changes of CT Findings in 90 Patients with COVID-19 Pneumonia: A Longitudinal Study. Radiolog 296(2).

10. Kuderer NM, Choueiri TK, Shah DP, Shyr Y, Rubinstein SM, et al. (2020) Clinical impact of COVID-19 on patients with cancer (CCC19): a cohort study. Lancet 395(10241): 1907-1918.

11. Hansell DM, Bankier AA, MacMahon H, McLoud TC, Müller NL, et al. (2008) Fleischner Society: Glossary of Terms for Thoracic Imaging. Radiology 246(3): 697-722.

12. Devresse A, Belkhi L, Vo B, Ghaye B, Scohy A, et al. (2020) COVID-19 Infection in Kidney Transplant Recipients: A Single-Center Case Series of 22 Cases From Belgium. Kidney Med 2(4): 459-466.

13. Simpson S, Kay FU, Abbara, S, Bhalla, S, Chung JH, et al. (2020) Radiological Society of North America Expert Consensus Statement on Reporting Chest CT Findings Related to COVID-19. Endorsed by the Society of Thoracic Radiology, the American College of Radiology, and RSNA. Radiology. Cardiothoracic Imaging 2(2): e200152.

14. Chung M, Bernheim A, Mei $X$, Zhang N, Huang $M$ et al. (2020) CT Imaging Features of 2019 Novel Coronavirus (2019-nCoV). Radiology 295(1): 202-207.

15. Bai HX, Hsieh B, Xiong Z, Halsey K, Choi JW, et al. (2020) Performance of radiologists in differentiating COVID-19 from viral pneumonia on chest CT. Radiology 296(2).

16. Wu J, Wu X, Zeng W, Guo D, Fang Z, et al. (2020) Chest CT Findings in Patients with Corona Virus Disease 2019 and its Relationship with Clinical Features. Invest Radiol 55(5): 257-261.

17. Wang Y, Dong C, Hu Y, Li C, Ren Q, et al. (2020) Temporal Changes of CT Findings in 90 Patients with COVID-19 Pneumonia: A Longitudinal Study. Radiology 296(2).

18. Liang W, Guan W, Chen R, Wang W, Li J, et al. (2020) Cancer patients in SARS-CoV-2 infection: a nationwide 
analysis in China. The Lancet Oncology 21(3): 335-337.

19. Guan WJ, Ni ZY, Hu Y, Liang WH, Ou CQ, et al. (2020) Clinical Characteristics of Coronavirus Disease 2019 in China. The New England journal of medicine. China Medical Treatment Expert Group for Covid-19 382(18): 1708-1720.

20. Chen N, Zhou M, Dong X, Qu J, Gong F, et al. (2020) Epidemiological and clinical characteristics of 99 cases of 2019 novel coronavirus pneumonia in Wuhan, China: a descriptive study. Lancet 395(10223): 507-513.

21. Zhang L, Zhu F, Xie L, Wang C, Wang J, et al. (2020) Clinical characteristics of COVID-19-infected cancer patients: a retrospective case study in three hospitals within Wuhan, China. Ann oncol 31(7): 894-901.

22. Ma J, Yin J, Qian Y, Wu Y (2020) Clinical characteristics and prognosis in cancer patients with COVID-19: A single center's retrospective study. The Journal of infection 81(2): 318-356.

23. Zhao W, Zhong Z, Xie X, Yu Q, Liu J (2020) Relation Between Chest CT Findings and Clinical Conditions of Coronavirus Disease (COVID-19) Pneumonia: A Multicenter Study. American Journal of Roentgenology 214(5): 1072-1077.

24. Desai A, Warner J, Kuderer N, Thompson M, Painter C, et al. (2020) Crowdsourcing a crisis response for COVID-19 in oncology. Nature cancer 1(4).

25. Lee L, Cazier JB, Starkey T, Turnbull CD, Kerr R, et al. (2020) COVID-19 mortality in patients with cancer on chemotherapy or other anticancer treatments: a prospective cohort study. Lancet 395(10241): 19191926.

\section{Clinical Radiology \& Imaging Journal}

26. Mohile S, Dumontier C, Mian H, Loh KP, Williams GR, et al. (2020) Perspectives from the Cancer and Aging Research Group: Caring for the vulnerable older patient with cancer and their caregivers during the COVID-19 crisis in the United States. Journal of geriatric oncology 11(5): 753-760.

27. Weinkove R, McQuilten ZK, Adler J, Agar MR, Blyth E, et al. (2020) Managing haematology and oncology patients during the COVID-19 pandemic: interim consensus guidance. The Medical journal of Australia 212(10): 481489.

28. Zu ZY, Jiang MD, Xu PP, Chen W, Ni QQ, et al. (2020) Coronavirus Disease 2019 (COVID-19): A Perspective from China. Radiology 296(2).

29. XieX,Zhong Z, Zhao W, Zheng C, Wang F, et al. (2020) Chest CT for Typical 2019-nCoV Pneumonia: Relationship to Negative RT-PCR Testing. Radiology 296(2).

30. Ai T, Yang Z, Hou H,Zhan C, Chen C, et al. (2020) Correlation of Chest CT and RTPCR Testing in Coronavirus Disease 2019 (COVID-19) in China: A Report of 1014 Cases. Radiology 296(2).

31. Pan Y, Guan H, Zhou S, Wang Y, Li Q et al. (2020) Initial CT findings and temporal changes in patients with the novel coronavirus pneumonia (2019-nCoV): a study of 63 patients in Wuhan, China. European Radiology 30(6): 3306-3309.

32. Trapani D, Marra A, Curigliano G (2020) The experience on coronavirus disease 2019 and cancer from an oncology hub institution in Milan, Lombardy Region. Eur J Cancer 132: 199-206. 